\title{
Digital fabrication strategies for timber thin-walled sections
}

\author{
Y. Al-Qaryouti, J. M. Gattas, R. Shi \& L. McCann \\ School of Civil Engineering, University of Queensland, Australia
}

\begin{abstract}
A rise in digital fabrication technologies has led to numerous recent advances in the design and manufacture of woodworking joints. Computational generative processes can be used to create a part with integral mechanical attachments for friction-fit, adhesion-less connections between parts. These processes can additionally create machine code for production of such parts on computernumerically controlled (CNC) cutters. This paper investigates such computational processes for digital design and manufacture of timber thin-walled structural sections. Assembly procedures are first presented for rectangular hollow sections and I-sections, with a series of timber prototypes produced to demonstrate their CNC production. An experimental analysis on square hollow sections under uniaxial compressive loading is then conducted. Tight fit digitally-fabricated sections are seen to possess a structural capacity approaching that of the glued sections. Prototypes with different assembly tolerances and grain directions produce a range of novel failure modes and give insight into potential failure paths of digitally-fabricated timber sections.
\end{abstract}

Keywords: digital fabrication, timber engineering, thin-walled sections.

\section{Introduction}

\subsection{Digital Fabrication}

The Digital Age is transforming how we design, build, and produce structures [1] with digital modelling and fabrication tools now available that provide a degree of flexibility and coordination never previously available [2]. Digital modelling tools such as computer-aided design (CAD) software are already widely available in design practice, however computer-aided manufacture (CAM) software and 
computer-numerical controlled $(\mathrm{CNC})$ manufacturing machines are increasingly also located in the hands of designers [3]. Digital fabrication is the broad term for procedural algorithms that can link $\mathrm{CAD} / \mathrm{CAM} / \mathrm{CNC}$ processes and thus integrate architectural design, engineering design, and manufacturing processes. For example, digital fabrication processes can subdivide a building envelope into elements suitable for fabrication from automated workshop machines such as CNC routers or laser cutters [4].

Sophisticated digital fabrication processes are also able to incorporate construction processes by considering means by which conventional mechanical fastening systems, such as screws and nails, can be eliminated from construction by inclusion of integral attachments in manufactured elements [5]. These are typically in the form of mechanical connections between components, for example through a fine control of part tolerance to achieve friction-only fit [6], or through interlocking geometry which prevent the movement of two parts in all but one direction and thus prevent disassembly [7]. Successful application of digital fabrication techniques enable extreme fabrication and assembly speeds, for example the 'Instant House' was assembled in 4 days from 984 plywood components [8] and the 'Plate House' was manufactured in 5 hours and assembled in 7 hours from 150 cardboard components [9].

\subsection{Modular timber construction}

Many of the above digitally-fabricated structures were constructed from timber, due to its high machinability, low weight, low assembly effort, and high sustainability credentials. More broadly, modular prefabricated timber construction is of increasing interest internationally for essentially the same reasons [10-12]. However, knowledge of the structural mechanics of timber structures and digitally-fabricated structures is still sparse compared to traditional structural materials and systems. Load tests on integral connections have shown that imperfections in the material and load distribution are critical to ultimate capacity [13]. Load testing of a digitally-fabricated arch made from $21 \mathrm{~mm}$ thick laminated-veneer lumber panels showed the arch was capable of sustaining a lineload up to $45 \mathrm{kN}$ and that joint rigidity was a critical factor in determining global stiffness [14].

The aim of the following paper is to develop a digital fabrication process for building elements, rather than an overall building surface as used in previous studies. Specifically, the present study is concerned with structural thin-walled sections (shown in fig. 1). Section 2 presents a digital fabrication process for rectangular hollow sections (RHS) and I-sections, encompassing a parametric definition of both section geometries, decomposition in 2D component plates, and superposition of integral mechanical connections. Friction-fit plywood prototypes are assembled to validate the method. Section 3 then presents an experimental investigation into a plywood square hollow sections under uniaxial compression, with loose fit, tight fit, and glued prototypes constructed and tested. A comparison of the measured capacities and observed failure modes is then presented in Section 4. 
a)

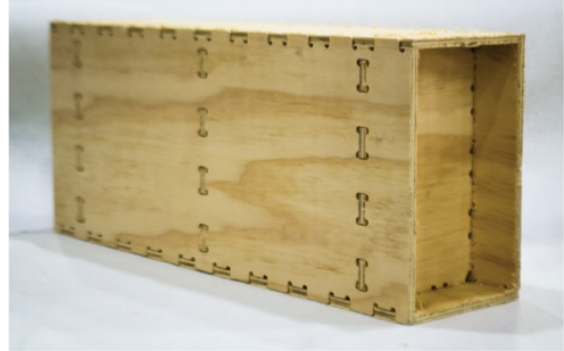

c)

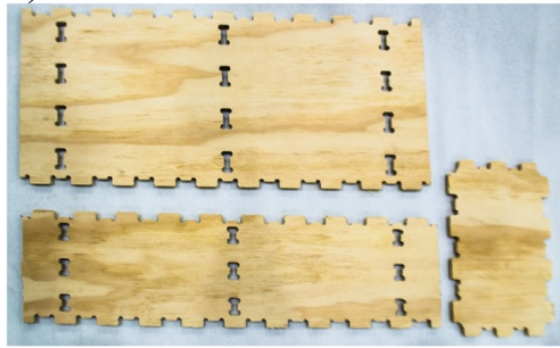

b)

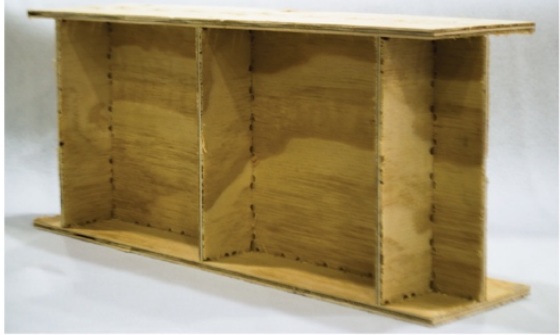

d)

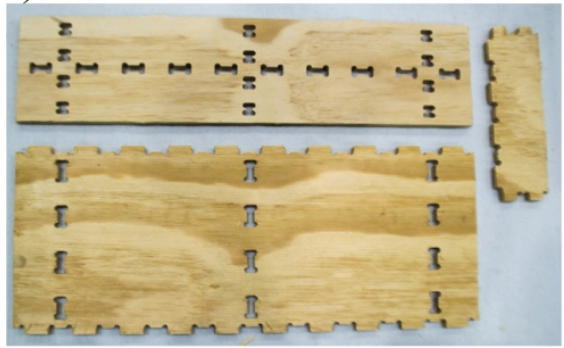

Figure 1: CNC-manufactured thin-walled a) RHS and b) I-sections. Component plates for each shown in c) and d), respectively.

\section{Digital fabrication of thin-walled sections}

\subsection{Parametric section definition}

Two common thin-walled sections, rectangular hollow sections and I-sections, are shown in fig. 2a) and b), respectively. Both are shown with bracing plates and can be parametrically defined with section depth $d$, section width $b$, wall thickness $t$, element length $L$, and brace plate locations $0<L_{i}<L$ where $L_{i}$ is the longitudinal position of the $i^{\text {th }}$ brace plate. If considered as an assemblage of $2 \mathrm{D}$ plate components, the RHS is composed of three unique plates: a web plate with area $L$ $\mathrm{x} d$, a flange plate with area $L \times b$, and a bracing plate with area $b \times d$, shown in fig. 2c). An I-section can similarly be decomposed into the three component plates as shown fig. $2 \mathrm{~d}$ ), except with bracing plate having an area $b / 2 \times d$.

\subsection{Integral connections}

The RHS and I-sections can be specified with the same control parameters, however differences are seen in the junctions between plates in the assembled section. Three junction types exist: web-flange; flange-brace; and web-brace junctions. There is variation in terms of required connectivity between different junction types. For example, the web-flange RHS junction is a 'corner' joint between web and flange plate edges, whereas the web-flange I-section junction is 
a)

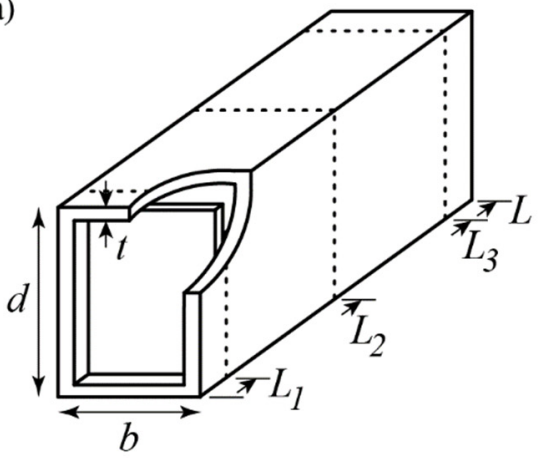

c)
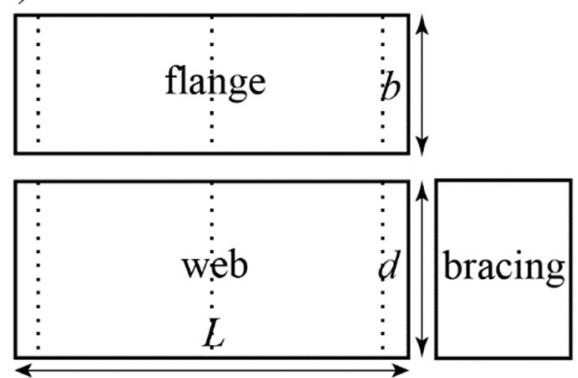

b)

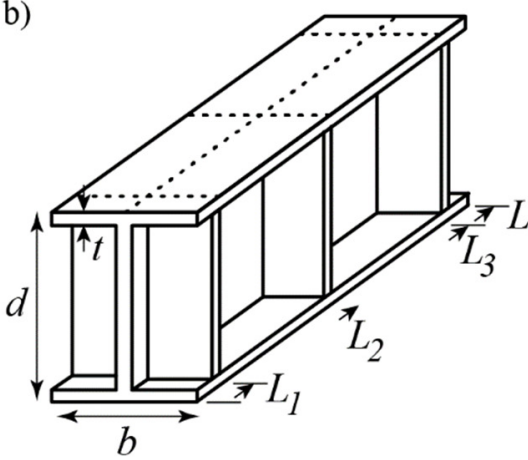

d)
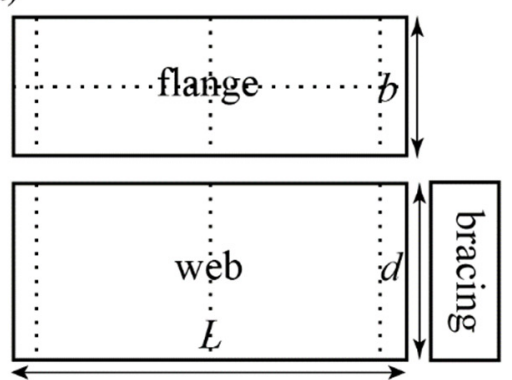

Figure 2: Parametric definition of a) RHS and b) I-section. Component plates for each shown in c) and d), respectively.

a 'tee' joint between the web edge and the flange centreline. Flange-brace and web-brace junctions for both section types are similarly a tee joint.

Appropriate integral connection detail for corner and tee joint types can also be parametrically specified. For a plywood corner joint, male and female connections with alternate tab locations are used, shown in fig. 3a) as connections $C_{1}$ and $C_{2}$, respectively. Control parameters include base tab width $w_{o}$, depth $d_{o}$, leading edge width $w_{l}$, and router bit diameter $d_{r}$, which is not shown for clarity but discussed further below. A tolerance parameter $\pm w_{t}$ is also used to oversize tabs to control the fit when assembled. For example, $\pm w_{t}=0$ will generate a tab the same width as its corresponding gap, whereas $\pm w_{t}=0.1 \mathrm{~mm}$ will generate a tab with a width $0.2 \mathrm{~mm}$ smaller than its corresponding gap. A tee connection is shown in fig. $3 \mathrm{~b}$ ), with a $C_{l}$ tab connection paired with a $C_{3}$ slot connection. Slot control parameters are the same as those listed above, except with the $\pm w_{t}$ tolerance undersizing, rather than oversizing, the slot width.

\subsection{Connection superposition}

The section and connection parameters are independent; however, they can be combined using a method developed by the second author for digital fabrication of plate-based sandwich structures [9]. First, the 2D section plate outlines are tagged with their junction type, for example an RHS web plate with a corner 
a)

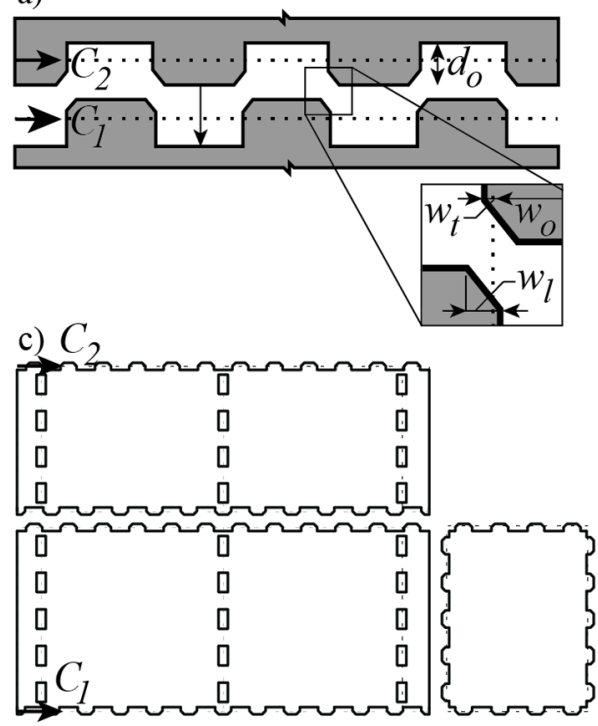

b)

d)
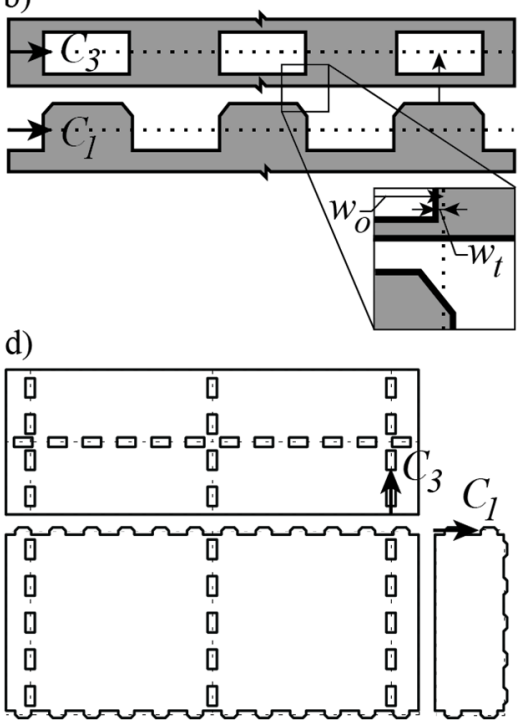

Figure 3: Parametric a) corner and b) tee connections. c) RHS and d) I-section component plates generated from integral connection superposition.

junction will have a $C_{1}$ tag along the top edge and the corresponding web plate will have a $C_{2}$ tag along the base edge. Similarly, plate edges for a tee junction will be tagged with $C_{I}$ and $C_{3}$ connection. Second, an assembler implemented in MATLAB [15] is used to scale and orient the connection detail to every tagged plate edge, generating a final part drawing for manufacture. Example tagged edges and generated part drawings are shown in fig. 3c) and d) for RHS and I-sections, respectively.

The part drawings are 2D and thus can be manufactured on any $\mathrm{CNC}$ cutter. The present method has been used with laser cutters, routers, and waterjet cutters. Example sections manufactured with a CNC router are shown in fig. 1, with both RHS and I-sections having section parameters $d=250 \mathrm{~mm}, b=150 \mathrm{~mm}, L=$ $500 \mathrm{~mm}$, and $t=9 \mathrm{~mm}$; and connection parameters $w_{o}=25 \mathrm{~mm}, d_{o}=t=9 \mathrm{~mm}, w_{l}=$ $2.5 \mathrm{~mm}, d_{r}=8 \mathrm{~mm}$, and $w_{t}= \pm 0.16 \mathrm{~mm}$. Note the router bit diameter parameter was not shown previously as it is not necessary for all processes. The pierce diameter for a laser cutter is approximately $0.3 \mathrm{~mm}$ and for a waterjet cutter approximately $0.8 \mathrm{~mm}$, which is small enough to generate sufficiently square internal corners for snug slot and tab pocket assembly. Router cutting diameter is dependant on the selected bit, but is typically at least $3 \mathrm{~mm}$. This leaves a radius when cutting internal corners which can prevent snug assembly. The router bit diameter parameter compensates for this by including semi-circular pockets at internal corners of $C_{1}, C_{2}$, and $C_{3}$ connections. 


\section{Experimental analysis}

\subsection{Method}

An experimental analysis was conducted to gain an insight into structural behaviours of digitally-fabricated plywood thin-walled sections. The present study restricts consideration to unbraced RHSs under uniaxial compression. Connection tolerance parameter $w_{t}$ and plywood grain direction were judged likely to be the critical factors on the peak compressive capacity of plywood thin-walled columns. Therefore, six different section designs were constructed for testing, consisting of three different connection types each with grain directions both parallel and perpendicular to the column axis and load direction. Two connection types were digitally-fabricated and relied on friction-fit corner junctions for structural cohesion: 'loose fit' which are pressed together by hand and 'tight fit' which are pressed together with a mallet. The third connection type is 'glued' and uses a loose fit part with Adheseal Unobond polyurethane adhesive applied along junctions prior to assembly.

\subsection{Fabrication}

Section parameters were selected as $d=b=168 \mathrm{~mm}, L=500 \mathrm{~mm}$, and $t=9 \mathrm{~mm}$, creating an unbraced square hollow section made of four identical plates. Connection parameters were selected as $w_{o}=25 \mathrm{~mm}, d_{o}=t=9 \mathrm{~mm}, w_{l}=2.5 \mathrm{~mm}$, and $d_{r}=0 \mathrm{~mm}$. Component parts were cut from grade C-D structural plywood with stress grade F11-F14, using a CNC waterjet cutter. 24 component plates could fit on a $2400 \mathrm{~mm}$ by $1200 \mathrm{~mm}$ plywood sheet which could be waterjet-cut in approximately 30 minutes, so a single column sample can be manufactured approximately every 5 minutes.

Connection tolerances for loose fit and tight fit models were selected following a calibration study. A series of $100 \mathrm{~mm} \times 100 \mathrm{~mm}$ square plates were manufactured with the above $C_{1}$ and $C_{2}$ connection parameters and with $w_{t}$ varied in $0.05 \mathrm{~mm}$ increments from $\pm 0.0 \mathrm{~mm}$ to $\pm 0.3 \mathrm{~mm}$. Tight fit tolerance was gained at $w_{t}=$ $\pm 0.2 \mathrm{~mm}$ and loose fit tolerance at $\pm 0.00 \mathrm{~mm}$. Samples with $w_{t}= \pm 0.25 \mathrm{~mm}$ and greater were too large to fit into the corresponding slot component without visible damage to tabs. The CNC waterjet software includes kerf compensation for the $0.8 \mathrm{~mm}$ cutting diameter and so manufactured tabs were found from measurement to be accurate to within $\pm 0.05 \mathrm{~mm}$ of drawn dimensions.

\subsection{Testing and results}

Three samples of each of the six column types were tested, with compression load applied to the samples at a rate of $1.0 \mathrm{~mm} /$ minute using an Intron Universal Testing Machine. Boundary conditions were set with a rigid base plate and a rigid top plate mounted on a spherical seat. Load-displacement data was recorded at the Instron cross head, with peak force values summarised in tab. 1 and plots shown in fig. 4. 
Table 1: Experimental specimen description and recorded peak loads.

\begin{tabular}{|c|c|c|c|c|c|c|c|c|c|}
\hline \multirow[t]{2}{*}{ Prototype } & \multirow{2}{*}{$\begin{array}{l}\text { Tolerance } \\
(\mathrm{mm}) / \\
\text { adhesive }\end{array}$} & \multicolumn{4}{|c|}{ Parallel grain $(\mathrm{kN})$} & \multicolumn{4}{|c|}{ Perpendicular grain $(\mathrm{kN})$} \\
\hline & & S1 & S2 & S3 & Avg. & S1 & $\mathrm{S} 2$ & S3 & Avg. \\
\hline Glued & \pm 0.00 /Unobond & 109.3 & 90.7 & 91.4 & 97.2 & 49.9 & 41.7 & 57.5 & 50.4 \\
\hline Tight Fit & \pm 0.20 /None & 97.9 & 81.5 & 85.7 & 88.4 & 49.7 & 43.9 & 54.7 & 48.8 \\
\hline Loose Fit & \pm 0.00 /None & 94.3 & 73.7 & 78.5 & 82.2 & 9.4 & 22.2 & 19.2 & 16.9 \\
\hline
\end{tabular}

a)

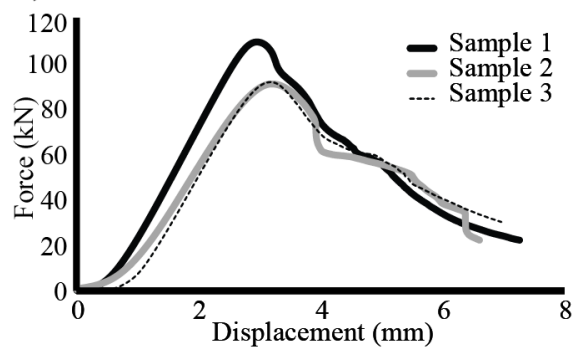

c)

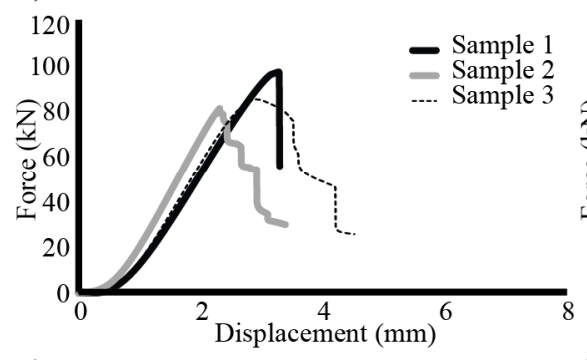

e)

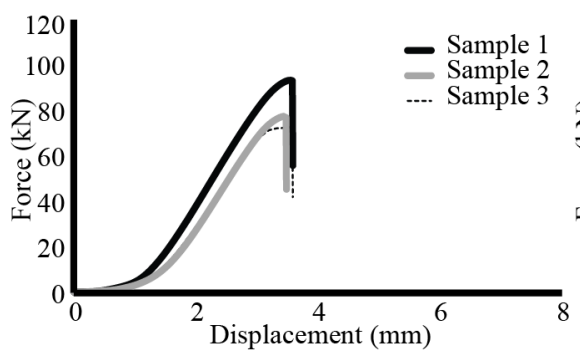

b)

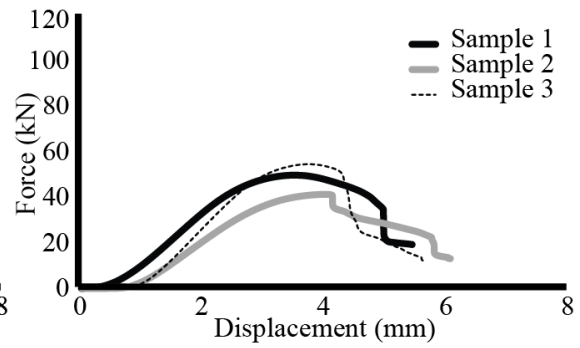

d)

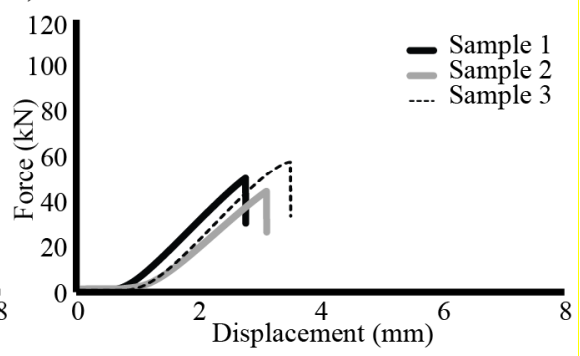

f)

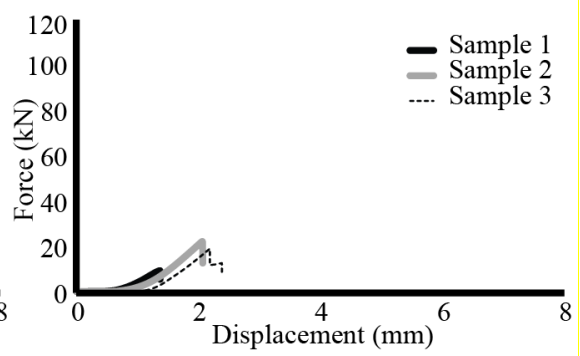

Figure 4: Load-displacement responses of tested specimens. On left, prototypes with grains parallel to the direction of loading: a) glued, c) tight fit, and e) loose fit. On right, prototypes with grains perpendicular to the direction of loading: b) glued, d) tight fit, and f) loose fit. 


\section{Discussion}

\subsection{Failure load}

Recorded peak force values for the six column types are summarised in fig. 5 . There is seen to be good consistency in peak failure loads between individual samples of the same type. All but one type have individual prototype values within $17 \%$ of averaged values, which is a variation comparable to natural material variation in plywood and the stress grade range specified by the plywood manufacturer. The exception is loose fit columns with a grain direction perpendicular to load. These samples have up to $45 \%$ variation from average, which is attributed to their particular failure mode and discussed further below. In comparing parallel and perpendicular grain samples, glued, tight fit, and loose fit parallel samples are $98 \%, 76 \%$, and $386 \%$ above their perpendicular equivalents, respectively. This is to be expected since the plywood panels loaded cross grain have significantly reduced compressive strength and flexural rigidity. The relation between this and observed failure modes is discussed further below.

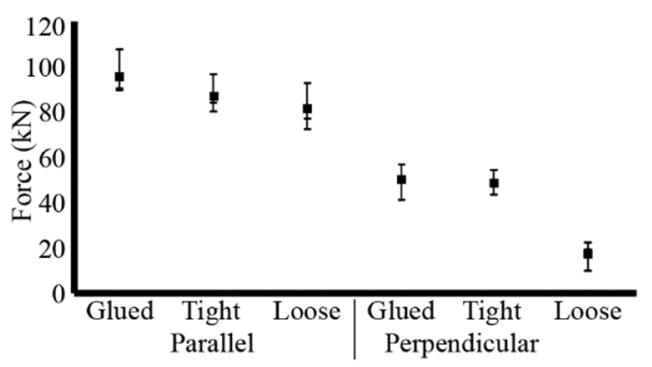

Figure 5: Summary of individual sample and average peak force values for each column type.

In comparing prototypes with different connection types, significant variation can be observed between sample sets. Glued samples exhibited stronger average compressive strengths than tight fit samples by just $10 \%$ for parallel grain samples and 3\% for cross grain samples. There was a larger difference compared with loose fit samples, with glued stronger than loose fit by $18 \%$ for parallel grain samples and $190 \%$ for perpendicular grain samples. The differences in peak load capacity between column types can be understood through inspection of failure modes.

\subsection{Failure modes}

A range of complex failure modes were exhibited across column types, with typical modes of each shown in fig. 6. Glued samples with grains parallel to loading were the only samples not to exhibit joint failure, but rather failed with local buckling and subsequent material fracture about column end regions as shown in fig. 6a). The failure location is likely attributable to bearing stress 
concentrations near boundary plates. Some samples also exhibited inter-veneer delamination at corner joints as failure progressed. Glued samples with grains perpendicular to load direction exhibited a similar end region buckling but with a subsequent loss of corner joint cohesion as shown in fig. 6b). Inspection of joints showed that failure was typically debonding or local plywood fracture at glued tabs.

Loose fit and tight fit samples with grains parallel to the direction of loading exhibited a 'pop-off' failure whereby one plate popped off the side of the prototype to form a column buckling mode. Adjacent plates formed a buckling failure mode typical for plates under uniaxial compression with three supported edges and one unsupported edge, shown in fig. 6c) and e). In tight fit prototypes, face pop-off was initially a slow, step-wise process in which tabs gradually separated at plate boundaries until a full plate pop-off. Inspection of plates after failure showed that tab separation was typically through delamination of the innermost plywood veneer layer and fracture across the tab. The popped-off plate often also showed cross-grain fracture. In loose fit samples, pop-off was almost instantaneous, with inspection of failed plates showing that plate boundaries failed with and without visible damage to tabs, implying that exceedance of friction cohesion capacity caused pop-off in some cases. Failure is therefore hypothesised to be a snapthrough between a square tube local buckling mode and a single-face column buckling mode, with the critical load governed by friction cohesion at plate boundaries and inter-ply delamination and fracture of tabs.

Loose fit and tight fit samples with grains perpendicular to the direction of loading exhibited a novel 'exploding barrel' failure whereby all component plates buckled outwards near-simultaneously, shown in fig. 6d) and f). Individual plates act as buckled columns post-failure and inspection of the plates after unloading showed no visible damage. The failure is therefore hypothesised to be a snapthrough between a square tube local buckling mode and a four-face column buckling mode, with the critical load governed by friction cohesion at plate boundaries. The column buckling mode is a function of stiffness and moment of inertia. Both are substantially reduced in plywood for cross-grain loading, particularly moment of inertia, as the contribution from the two outermost ply layers is neglected as their grain is perpendicular to load direction. This means the loose fit columns loaded perpendicular have minimal frication cohesion to resist a snap to weak column buckling mode which could cause the high variability seen between sample peak loads.

To summarise, a range of complex modes were exhibited with relatively minor variations in column geometry and construction. Despite this, digitally-fabricated samples were seen to almost match the capacity of glued samples, and are likely to be capable of further improvements given the above insights into potential failure paths. Ongoing work is underway to more precisely characterise material and assembly type failure modes to better control such behaviours. 
a)

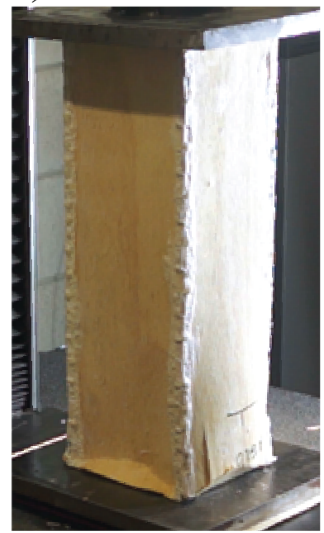

c)

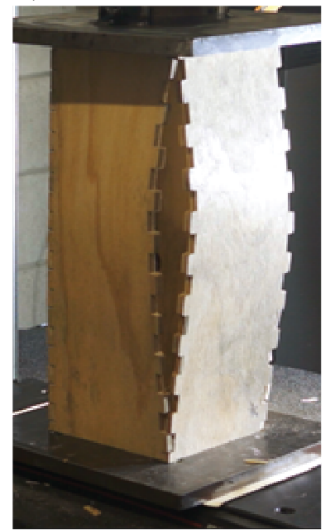

e)

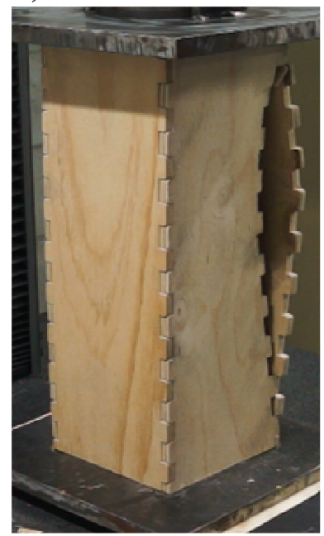

b)
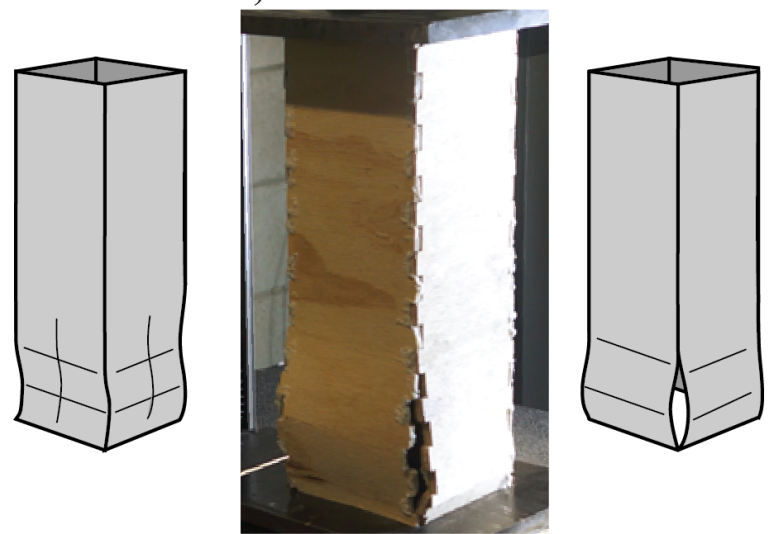

d)
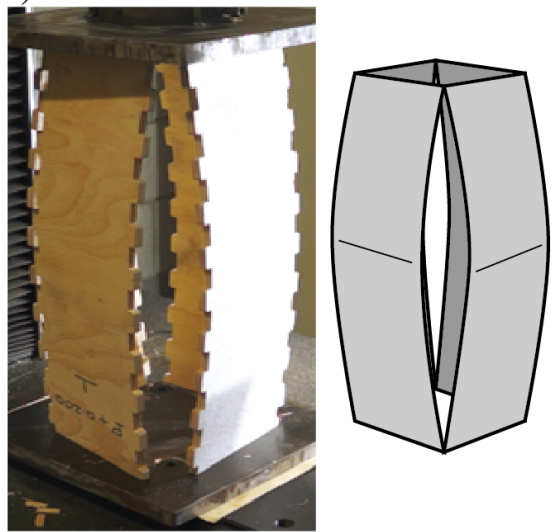

f)
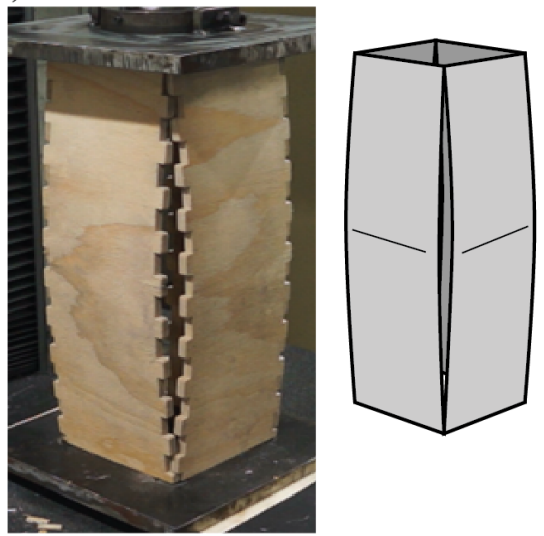

Figure 6: Failure modes of tested specimens. On left, prototypes with grains parallel load: a) glued, c) tight fit, and e) loose fit. On right, prototypes with grains perpendicular load: b) glued, d) tight fit, and f) loose fit. 


\section{Conclusion}

This paper investigated computational generative processes for digital design and fabrication of plywood thin-walled structural sections. Fabrication principles and techniques were presented for both rectangular hollow sections and I-sections and validated with a series of CNC-manufactured prototypes to demonstrate the novel form of production.

Experimental tests were conduction on square hollow sections under uniaxial compressive loading to compare glued sections with loose fit and tight fit digitallyfabricated sections. Tests showed that the tight fit sections have a structural compressive strength close to the conventional glued sections for loading both parallel and perpendicular to plywood grain direction. However, a wide range of complex failure modes were exhibited between column types with relatively minor variations in geometry and construction, including square tube buckling, ply and adhesive delamination, column and plate buckling, and partial and complete face pop-off. Results give a strong insight into potential failure paths of digitallyfabricated timber sections and ongoing work is underway to characterise these failures to better design such structures.

\section{Acknowledgements}

The authors would like to acknowledge undergraduate research students Harry Pagliaro and Fei Zhou for their assistance with design and manufacture of prototypes. The first author is also grateful for the financial support from Australian Research Council Discovery Early Career Research Award DE160100289.

\section{References}

[1] Kolarevic, B., Digital Fabrication: Manufacturing Architecture in the Information Age. In Proceedings of the 21st Annual Conference of the Association for Computer-Aided Design in Architecture, Buffalo (New York), pp. 268-278, 2001.

[2] Iwamoto, L, Digital Fabrication: Architectural and Material Techniqures, New York: Princeton Architectural Press, 2009.

[3] Gu, N. \& Wang, X., (eds). Computational Design Methods and Technologies: Applications in CAD, CAM and CAE Education: Applications in CAD, CAM and CAE Education, IGI Global, 2012.

[4] Gramazio, F. \& Kohler, M, Digital Materiality in Architecture, Baden: Lars Müller Publishers, 2008.

[5] Genc, S., Messler Jr, R.W. \& Gabriele, G.A, A Systematic Approch to Integral Snap-fit Attachment Design. Research in Engineering Design 10(2), pp. 84-93, 1998.

[6] Sass, L., Mega Assembly: Scaling \& Decomposition of Digital Designs. Presented at The Science of Digital Fabrication workshop, MIT, 2013. 
[7] Robeller, C, Stitic, A, Mayencourt, P \& Weinland, Y, Interlocking Folded Plate - Integrated Mechanical Attachment for Structural Wood Panels. Advances in Architectural Geometry, vol. 4, pp. 281-294, 2014.

[8] Sass, L \& Botha, M, The Instant House: A Model of Design Production with Digital Fabrication. International Journal of Architecutral Computing, vol. 4, pp. 109-123, 2006.

[9] Gattas, J.M \& You, Z, Design and Digital Fabrication of Folded Sandwich Structures. Automation in Construction, pp. 63, 79-87, 2016.

[10] Lawson, M, Odgen, R. \& Goodier, C., Design in Modular Construction, CRC Press, 2014.

[11] Smith, I \& Snow, M, Timber: An Ancient Construction Material with a Bright Future. Forestry Chronicle, vol. 4, no. 84, pp. 504-510, 2008.

[12] Li, R. Y. M. \& Du H., Sustainable Construction Waste Management in Australia: A Motivation Perspective. Construction Safety and Waste Management, Springer International Publishing, pp. 1-30, 2015.

[13] Wagner, M. E., Structural connections in plywood friction-fit construction, Doctoral dissertation, Massachusetts Institute of Technology, 2014.

[14] Robeller, C., Stitic, A., Mayencourt, P. \& Weinand, Y., "Interlocking Folded Plate: Integrated Mechanical Attachment for Structural Wood Panels. Advances in Architectural Geometry 2014, Springer International Publishing, pp. 281-294, 2015.

[15] Gattas, J., Digital fabrication toolbox. Available from: http://joegattas.com/digital-fabrication-toolbox, 2014. 